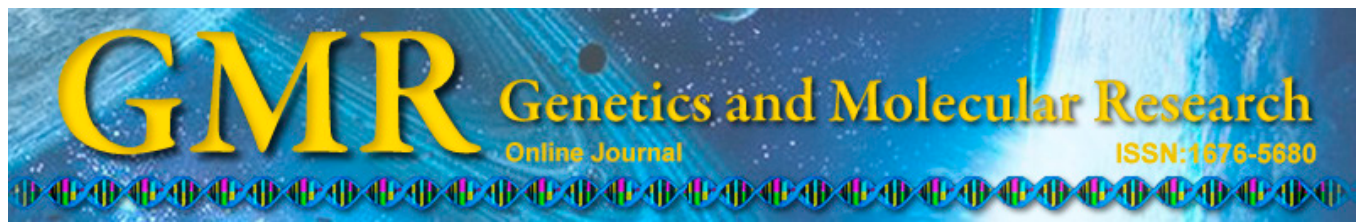

\title{
Molecular genetic analysis of heterosis in interspecific hybrids of Argopecten purpuratus $\mathbf{x}$ A. irradians irradians
}

\author{
L.P. Hu ${ }^{1,2}$, X.T. Huang ${ }^{1}$, Y. Sun ${ }^{1}$, J.X. Mao', S. Wang ${ }^{1}$, C.D. Wang ${ }^{3}$ and \\ Z.M. Bao ${ }^{1}$
}

${ }^{1}$ Key Laboratory of Marine Genetics and Breeding, Ministry of Education, Ocean University of China, Qingdao, Shandong, China

${ }^{2}$ Yantai Fisheries Research Institute, Yantai, Shandong, China

${ }^{3}$ College of Marine Science and Engineering, Qingdao Agricultural University,

Qingdao, Shandong, China

Corresponding author: X.T. Huang

E-mail: xthuang@ouc.edu.cn

Genet. Mol. Res. 14 (3): 10692-10704 (2015)

Received December 9, 2014

Accepted May 15, 2015

Published September 9, 2015

DOI http://dx.doi.org/10.4238/2015.September.9.9

\begin{abstract}
Argopecten purpuratus and Argopecten irradians irradians hybridization was successfully performed and the hybrid offspring displayed apparent heterosis in growth traits. To better understand the genetic basis of heterosis, the genomic composition and genetic variation of the hybrids were analyzed with amplified fragment length polymorphism (AFLP) and simple sequence repeat (SSR) markers. Seven of eight universal SSR primers displayed polymorphism in the hybrids and their parental groups, and hybrids inherited both parental genotypes at each locus. Using five primer combinations in AFLP analysis, 433 loci were amplified in the hybrids and their parental groups. The frequency of polymorphisms was $88.22 \%$. F1 hybrids inherited 88.11 and $92.88 \%$ of AFLP bands from their parents. Some loci did not follow Mendelian Law, including 48 loci in parents that were lost, and 11 new loci that were amplified in the hybrids. The parameters of Nei's gene di-
\end{abstract}


versity, Shannon's Information index, genetic distance, and molecular variance between groups were calculated. The genetic differentiation between two hybrid groups $(0.253)$ was smaller than that between hybrids and their parents ( 0.554 to 0.645$)$, and was especially smaller than that between two parental groups $(0.769)$. The high genetic similarity $(0.9347)$ and low genetic differentiation $(0.2531)$ between two hybrid groups suggests that these hybrid groups were genetically very close. Heterozygosities of hybrid groups were higher than those of parental groups, indicating that the hybrids had increased genetic diversity.

Key words: AFLP; Argopecten; Heterosis; Hybrid; SSR

\section{INTRODUCTION}

Crossbreeding is an important strategy for breeding and producing heterosis, which has been widely attempted and utilized in many commercial species in aquaculture (Beck and Bigger, 1982; Yan et al., 1999; Hedgecock and Davis, 2000). In scallops, it has been widely tested and shown to be effective at improving disease resistance and productivity. For example, hybrid scallops (Patinopecten yessoensis x Patinopecten caurinus) showed resistance to Perkinsus qugwadi, which is a lethal pathogen of P. yessoensis (Bower et al., 1997). The hybrids of two stocks of the catarina scallop (Argopecten circularis) displayed strong maternal effects and heterosis in terms of growth and survival (Cruz and Ibarra, 1997). In China, an interspecific cross between Chlamys farreri and P. yessoensis was attempted, and their hybrid descendants showed greater genetic diversity than the parental populations based on random amplified polymorphic DNA (RAPD) analysis (Teng et al., 2005), and heterosis was displayed in both growth and disease resistance (Yang et al., 2004). Considering these data, interspecific hybridizations could provide an efficient way of utilizing heterosis and a potential approach of breeding excellent scallop varieties.

The bay scallop, Argopecten irradians irradians, which is distributed from the east coast of the United States to the Gulf of Mexico (Waller, 1969), was introduced to China from the USA in 1982 (Zhang et al., 1986) and soon became widely cultivated in northern China. The Peruvian scallop, Argopecten purpuratus, is naturally distributed along the Pacific coast of South America (Dall, 1909). Although these two scallop species are hermaphroditic Argopecten and have similar karyotypes (Wang and Guo, 2004), their size, life span, and temperature tolerance are distinct (Wang et al., 2011). These species may represent a good model system for crossbreeding to produce heterosis. The Peruvian scallop was introduced into China in 2007 and 2008, and was successfully hybridized with the bay scallop (Wang et al., 2011). The hybrid offspring showed an apparent increase in production characteristics and displayed interesting new traits. Hu et al. (2013) confirmed that the hybrids possessed 32 chromosomes, and by using genomic in situ hybridization, these were found to be a combination of haploid genomes from two parents. However, little is known about the genetic variation and diversity of these hybrids at the molecular level.

Molecular marker-assisted selection is expected to increase the speed and precision by which scallop breeding processes integrate desired characteristics from native varieties into elite varieties. DNA fingerprinting was first described by Jeffreys et al. (1985) and is now commonly used to study genetic variability and to analyze pedigree relationships in a wide 
variety of organisms including scallops (Song et al., 2002; Teng et al., 2005). Amplified fragment length polymorphism (AFLP) could allow high-resolution genotyping of fingerprinting quality. Microsatellites [simple sequence repeats (SSRs)] are highly variable and co-dominant markers. These two methods are thought to be efficient and reliable DNA markers, compared with RAPD and RFLP (Vos et al., 1995; Powell et al., 1996).

In the present study, AFLP and SSR were adopted to analyze the genetic constitution of four populations of scallops (A. purpuratus, A. irradians irradians, and their hybrids) at the molecular level, to detect the genetic variation and inheritance from the parents to F1 progeny, and to compare the genetic diversity of the hybrid and parental populations.

\section{MATERIAL AND METHODS}

\section{Scallop materials}

A. purpuratus (PP) and A. irradians irradians (BB) were selected as parental populations. Scallops were induced to spawn, and insemination was performed following the method outlined by Wang et al. (2011). The resulting hybrid larvae (A. purpuratus + x A. irradians irradians $\widehat{\partial}$, $\mathrm{PB}$ and $A$. irradians irradians ㅇ $\mathrm{x} A$. purpuratus $\widehat{\partial}$, $\mathrm{BP}$ ) were reared following routine culture procedures, as described by Zhang et al. (2007). The adult hybrid scallops were obtained after 6 months. The parental scallops and hybrids were collected from the Jiaonan scallop hatchery in Qingdao, Shandong Province, China. In total, 20 individuals from each group were randomly selected for analysis.

\section{DNA extraction}

Adult specimens were used for DNA preparation. The adductor muscles were removed from live individuals and stored in liquid nitrogen until use. DNA was extracted according to the traditional phenol/chloroform extraction method (Sambrook et al., 1989).

\section{AFLP analysis}

AFLP analysis was performed according to the protocol described by Vos et al. (1995), with minor modifications. Briefly, total genomic DNA was digested with EcoRI and MseI. Specific double-stranded adapters were subsequently ligated to the restriction fragment ends. The ligation products were then pre-amplified using EcoRI and MseI primers with one selective nucleotide at the $3^{\prime}$ end and then amplified again using primers with three selective bases at the 3' end. The structure of the adapter sequences was: EcoRI: 5'-CTCGTAGACTGC GTACC-3' and 3'-CTGACGCATGGTTAA-5'; MseI: 5'-GACGATGAGTCCTGAG-3' and 3'-TACTCAGGACTCAT-5'.

The primers were employed for preamplification and selective amplification with the following extensions: AAC/CAG (E32M49), AAC/CCT (E32M54), ACA/CTG (E35M61), ATC/CTG (E44M61), and AAG/CCT (E33M54). Amplified fragments were separated using $6 \%$ denaturing polyacrylamide gels (19:1 acrylamide: bis-acrylamide, $7 \mathrm{M}$ urea, $1 \mathrm{X}$ TBE buffer) and visualized via silver staining according to the method described by Sambrook et al. (1989). 


\section{Statistical analysis}

The silver stained AFLP bands in each gel were recorded as "1" to indicate the presence of a band or "0" to indicate no band. A binary data matrix was obtained. The following indices were calculated using POPGENE version 1.31 (Yeh et al., 1999): the number of polymorphisms, the assay efficiency index (Shannon's index), the gene diversity $(h)$ index, Nei's (1978) genetic similarity coefficient, and the standard genetic distance. The relationship between four populations was assessed with Nei's genetic distance between all pairs of populations using the UPGMA clustering method, which was modified from the NEIGHBOR procedure in PHYLIP Version 3.5. Molecular variance of the parents and the hybrids were assayed using analysis of molecular variance (AMOVA) (Arlequin 3.01) (Schneider et al., 2000).

\section{SSR analysis}

A total of 31 microsatellite markers developed for the bay scallop (referred to Roberts et al., 2005; Zhan et al., 2005, 2006) were assessed in Peruvian scallop to select the common markers. The chosen markers were then used for SSR analysis in the hybrids and parental groups. The annealing temperature for these analyses ranged from $50^{\circ}$ to $65^{\circ} \mathrm{C}$. PCR amplification was performed according to the protocol described by Zhan et al. (2005). PCR products were detected by electrophoresis on $10 \%$ non-denaturing polyacrylamide gel in $1 \mathrm{X}$ TBE buffer. The gels were stained with ethidium bromide and visualized under ultraviolet light (Zhan et al., 2005). The genotypes of different loci were scored with the software QUANTITY ONE version 4.4 (Bio-Rad) by comparing with the DNA molecular standard (100-bp DNA ladder marker, TaKaRa).

The level of genetic diversity per group was evaluated by the number of alleles $(\mathrm{N})$ and the effective number of alleles per locus $\left(A_{\mathrm{E}}\right)$, as well as the observed $\left(H_{\mathrm{O}}\right)$ and expected $\left(H_{\mathrm{E}}\right)$ heterozygosities using POPGENE version 1.31 (Yeh et al., 1999). The polymorphic information content (PIC) value was estimated according to the following formula:

$$
P I C=1-\left(\sum_{i=1}^{n} p_{i}^{2}\right)-\left(\sum_{i=1}^{n-1} \sum_{j=i+1}^{n} 2 p_{i}^{2} p_{j}^{2}\right)
$$

where $n$ is the allele number, and $P_{i}$ and $P_{j}$ are the frequencies of the $i^{\text {th }}$ and $j^{\text {th }}$ alleles.

\section{RESULTS}

\section{Characteristics of AFLP and SSR loci in the hybrids and their parents}

In AFLP analysis, five primer combinations revealed a total of 433 loci ranging in size from 50 to $1500 \mathrm{bp}$ in the hybrids and their parental groups, of which 382 were polymorphic. There was no significant difference in the number of amplified loci observed between these primer combinations. The number of amplified loci and the frequency of polymorphisms in each group are shown in Table 1. 


\begin{tabular}{|c|c|c|c|c|c|}
\hline & PP & PB & $\mathrm{BP}$ & BB & Total \\
\hline Number of amplification loci & 286 & 342 & 361 & 309 & 433 \\
\hline Number of polymorphic loci & 132 & 145 & 160 & 181 & 382 \\
\hline Frequency of polymorphism & $30.48 \%$ & $33.49 \%$ & $36.95 \%$ & $41.80 \%$ & $88.22 \%$ \\
\hline Specific amplification loci & 30 & 4 & 7 & 18 & - \\
\hline Percentage of specific loci & $10.49 \%$ & $1.17 \%$ & $1.94 \%$ & $5.83 \%$ & - \\
\hline
\end{tabular}

The representative AFLP amplification loci in hybrids are shown in Figure 1. In summary, the loci could be described as the following: 1) Loci were shared with both parents; they were not polymorphic among the four groups, with 161 of such loci, e.g., locus "a" in Figure $1 ; 2)$ loci were shared with one parent, including those shared with PP and with BB. Such loci were the most prevalent, and 252 and 287 were shared with PP and BB, respectively, e.g., the "b" and "c" loci shown in Figure 1; 3) loci were only presented in progeny, such as four loci in PB and seven loci in BP, which were not observed in any PP or BB individuals, and could be used to identify hybrids, e.g., the "d" shown in Figure 1; and 4) loci were present only in the parents and are likely to have been lost in the progeny. There were 48 such loci, e.g., the "e" locus shown in Figure 1. The inheritance of AFLP loci from the parents to F1 progeny was examined (Table 2), and $92.88 \%$ of amplified loci from BB and $88.11 \%$ of loci from PP were present in hybrid offspring.

Table 2. Transmission statistics of AFLP amplified loci from the parents to the F1 progeny.

\begin{tabular}{lcccccc}
\hline & PP-PB & PP-BP & PP-(PB\&BP) & BB-PB & BB-BP & BB-(PB\&BP) \\
\hline Number of transmitted markers & 239 & 241 & 252 & 262 & 278 & 287 \\
Percentage of transmitted markers (\%) & 83.57 & 84.27 & 88.11 & 84.79 & 89.97 & 92.88 \\
\hline
\end{tabular}

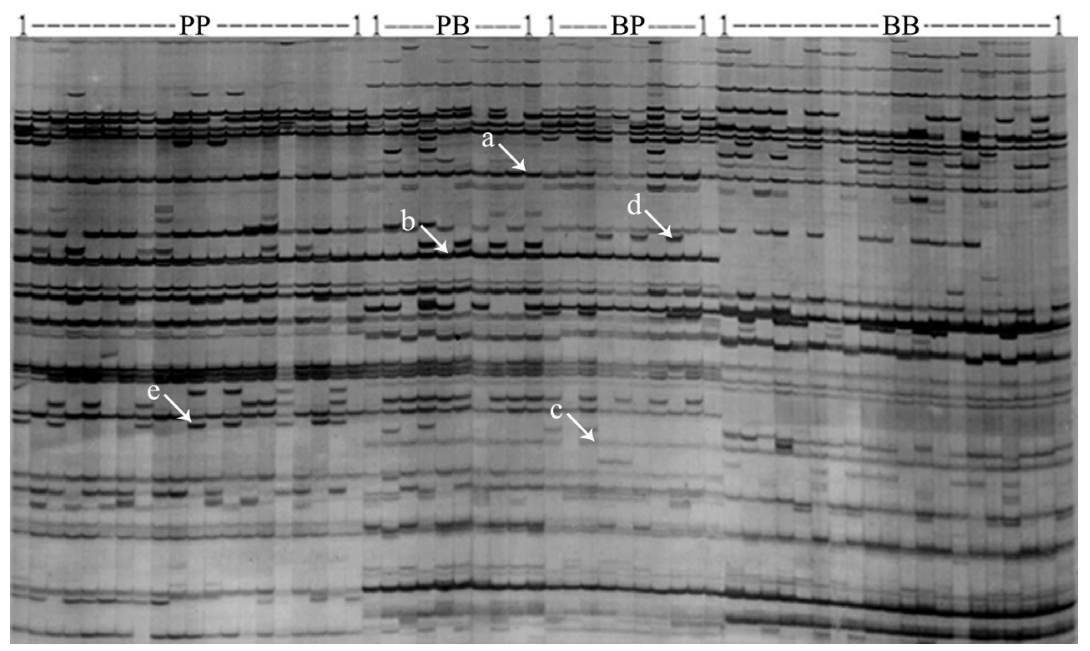

Figure 1. AFLP bands of Argopecten purpuratus (PP), Argopecten irradians (BB) and their hybrids of A. purpuratus

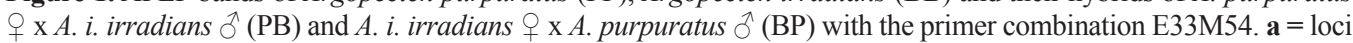
shared in hybrids (PB and BP) and their both parents (PP and BB); $\mathbf{b}=$ loci shared in hybrids and $\mathrm{PP} ; \mathbf{c}=$ loci shared in hybrids and $\mathrm{BB} ; \mathbf{d}=$ loci only presented in hybrid progeny; $\mathbf{e}=$ loci presented only in parents and likely lost in progeny. 
The transferability analysis of 31 microsatellite markers from the Bay scallop, $A$. irradians irradians revealed that eight primer pairs could produce amplified bands in the Peruvian scallop, A. purpuratus. These eight microsatellite markers were adopted to further analyze the genetic constitution of hybrids. The characteristics of these microsatellite markers are presented in Table 3. The amplification information of all SSR loci in four groups is shown in Table 4. For all groups (PP, BB, PB, and BP), 35 different alleles were found over all loci, ranging from one to eight alleles per locus. Of these, BB displayed 27 alleles for the eight loci, with a mean value of 3.38 and a mean allelic richness $\left(A_{\mathrm{E}}\right)$ of 2.5. PP only produced nine alleles over all loci. In F1 hybrids (PB and BP), 24 alleles appeared across the eight loci, and the number of alleles per locus ranged from one to six. The mean $A_{\mathrm{E}}$ was 2.2708 for $\mathrm{PB}$ and 2.3028 for BP, respectively. Moreover, except for locus AIMS007, which displayed no polymorphism in the four groups, the other seven loci presented different degrees of polymorphism. For the seven polymorphic loci, the sizes of alleles per locus in PP were different from those in BB. In the hybrids, two alleles per locus were amplified for these seven loci, of which, one appeared only in PP, and the other presented only in BB. Such as the amplified bands with the primers N391 in Figure 2, only one band of 221 bp was amplified in all PP individuals but not in any BB individual. On the contrary, four other bands of 226, 230, 232 (not shown in Figure 2), and $243 \mathrm{bp}$ were amplified in BB individuals. In all hybrids, two bands were amplified, one was the 221-bp band that was only observed in the PP and another that was observed only in BB. These data indicate that the hybrid offspring inherited genetic markers from both parental groups. The seven polymorphic loci could be developed as useful markers for identifying hybrids.

\begin{tabular}{|c|c|c|c|c|}
\hline Locus name & Accession No. & Primer sequences $\left(5^{\prime} \rightarrow 3^{\prime}\right)$ & $\mathrm{Ta}$ & Reference \\
\hline \multirow[t]{2}{*}{ AIMS001 } & CF197476 & F: TTCCTAATGGTGCGGGCTAC & & \\
\hline & & R: CATCATCGTACTCCTGGTTATC & 61 & Zhan et al. (2005) \\
\hline \multirow[t]{2}{*}{ AIMS007 } & CK484160 & F: TGTCAGAGTTCACAGCTAGGTGACC & & \\
\hline & & R: GGTTTCTCCTTGTTGTGGTCTGG & $50-57$ & Zhan et al. (2005) \\
\hline \multirow[t]{2}{*}{ AIMS021 } & CB415895 & F: GACGATGTGTTAGCTATTCAGAATC & & \\
\hline & & R: AGAGAAATTGTTGTTCGAGAGC & 56 & Zhan et al. (2006) \\
\hline \multirow[t]{2}{*}{ AIMS028 } & CB412414 & F: ATCTTATCCTGTGCCATTGGAC & & \\
\hline & & R: CTAAATCCTGAAACAAGATGCC & 63 & Zhan et al. (2006) \\
\hline \multirow[t]{2}{*}{ M26 } & CV660848 & F: CACTTTCAGCAGATATTCTTGAGG & & \\
\hline & & R: TCCCATCCTCTCCTTCACAG & 55 & Roberts et al. (2005) \\
\hline \multirow[t]{2}{*}{ GP63 } & CK484125* & F: AACTTTTCCCTCATCGTGTCACC & & \\
\hline & & R: CAGTCACAACTATCAACCTGCCC & 54 & Roberts et al. (2005) \\
\hline \multirow[t]{2}{*}{ N391 } & CN782436 & F: TCATCGCCTCCACCTTCAG & & \\
\hline & & R: GATCACACTTTGATTTGTCCTACG & 58 & Roberts et al. (2005) \\
\hline \multirow[t]{2}{*}{ S336 } & CN783139 & F: GCGGAGGCAGATTCTTTCTTTTC & & \\
\hline & & R: GGTCGTGGATTGTAAGCATTGTC & 54 & Roberts et al. (2005) \\
\hline
\end{tabular}

\section{Genetic variations in the hybrids and their parents}

In AFLP analysis, 421 loci were produced in PP and BB, and 247 loci, accounting for $58.67 \%$, were not shared between these two parental groups. Of these, 112 loci were amplified only in PP, and 135 were amplified only in BB, indicating that these loci could 
be used to identify the two groups. AMOVA was used to determine the genetic variations of $\mathrm{PP}$ and $\mathrm{BB}$ and the differentiation between them (Table 5). A large proportion of the variance $(76.85 \%)$ was observed between groups ( $\mathrm{PP}$ and $\mathrm{BB})$, and the genetic differential index (FST) between the groups was 0.769 . The genetic variations of PP and BB were significantly different $(\mathrm{P}<0.001)$ as indicated by a random permutation test. In contrast to the parental groups (PP and BB), AMOVA indicated that the genetic differentiation between $\mathrm{PB}$ and $\mathrm{BP}(0.2531)$ was very small, and the major portion of variance in $\mathrm{PB}$ and BP was within groups $(74.69 \%)$ (Table 5$)$.

Table 4. Genetic structure information revealed by microsatellites in the four populations.

\begin{tabular}{|c|c|c|c|c|c|c|}
\hline \multirow[t]{2}{*}{ Locus } & \multirow[t]{2}{*}{ Genetic indexes } & \multicolumn{4}{|c|}{ Populations } & \multirow[t]{2}{*}{ Total } \\
\hline & & PP & PB & $\mathrm{BP}$ & BB & \\
\hline AIMS001 & $\begin{array}{l}\mathrm{N}\left(A_{\mathrm{E}}\right) \\
H_{\mathrm{O}} \\
H_{\mathrm{E}} \\
\mathrm{PIC}\end{array}$ & $\begin{array}{c}1(1.0000) \\
0.0000 \\
0.0000 \\
0.0000\end{array}$ & $\begin{array}{c}3(2.5714) \\
1.0000 \\
0.6471 \\
0.5429\end{array}$ & $\begin{array}{c}4(2.9691) \\
1.0000 \\
0.6920 \\
0.6152\end{array}$ & $\begin{array}{c}3(2.5772) \\
0.0417 \\
0.6250 \\
0.5429\end{array}$ & $\begin{array}{c}4(3.5373) \\
0.3860 \\
0.7236 \\
0.6676\end{array}$ \\
\hline AIMS021 & $\begin{array}{l}\mathrm{N}\left(A_{\mathrm{E}}\right) \\
H_{\mathrm{O}} \\
H_{\mathrm{E}} \\
\mathrm{PIC}\end{array}$ & $\begin{array}{c}2(1.5355) \\
0.4500 \\
0.3577 \\
0.2879\end{array}$ & $\begin{array}{c}4(2.5474) \\
1.0000 \\
0.6364 \\
0.7296\end{array}$ & $\begin{array}{c}5(3.0250) \\
1.0000 \\
0.7013 \\
0.6260\end{array}$ & $\begin{array}{c}6(4.2667) \\
0.8333 \\
0.7819 \\
0.7296\end{array}$ & $\begin{array}{c}8(4.4223) \\
0.7727 \\
0.7798 \\
0.7491\end{array}$ \\
\hline AIMS028 & $\begin{array}{l}\mathrm{N}\left(A_{\mathrm{E}}\right) \\
H_{\mathrm{O}} \\
H_{\mathrm{E}} \\
\mathrm{PIC}\end{array}$ & $\begin{array}{c}1(1.0000) \\
0.0000 \\
0.0000 \\
0.0000\end{array}$ & $\begin{array}{c}2(2.0000) \\
1.0000 \\
0.5263 \\
0.5490\end{array}$ & $\begin{array}{c}3(2.4615) \\
0.7500 \\
0.6786 \\
0.5112\end{array}$ & $\begin{array}{c}3(2.6376) \\
0.7273 \\
0.6353 \\
0.5490\end{array}$ & $\begin{array}{c}4(3.1041) \\
0.5918 \\
0.6848 \\
0.6180\end{array}$ \\
\hline GP63 & $\begin{array}{l}\mathrm{N}\left(A_{\mathrm{E}}\right) \\
H_{\mathrm{O}} \\
H_{\mathrm{E}} \\
\mathrm{PIC}\end{array}$ & $\begin{array}{c}1(1.0000) \\
0.0000 \\
0.0000 \\
0.0000\end{array}$ & $\begin{array}{c}2(2.0000) \\
1.0000 \\
1.0000 \\
0.2609\end{array}$ & $\begin{array}{c}2(2.0000) \\
1.0000 \\
1.0000 \\
0.375\end{array}$ & $\begin{array}{c}2(1.4459) \\
0.2857 \\
0.3159 \\
0.2609\end{array}$ & $\begin{array}{c}3(2.4712) \\
0.1795 \\
0.6031 \\
0.5081\end{array}$ \\
\hline M26 & $\begin{array}{l}\mathrm{N}\left(A_{\mathrm{E}}\right) \\
H_{\mathrm{O}} \\
H_{\mathrm{E}} \\
\mathrm{PIC}\end{array}$ & $\begin{array}{c}1(1.0000) \\
0.0000 \\
0.0000 \\
0.0000\end{array}$ & $\begin{array}{c}3(2.6667) \\
1.0000 \\
0.6667 \\
0.6586\end{array}$ & $\begin{array}{c}2(2.0000) \\
1.0000 \\
0.5263 \\
0.375\end{array}$ & $\begin{array}{c}5(3.4184) \\
0.4583 \\
0.7225 \\
0.6574\end{array}$ & $\begin{array}{c}6(3.4429) \\
0.4677 \\
0.7153 \\
0.6746\end{array}$ \\
\hline N391 & $\begin{array}{l}\mathrm{N}\left(A_{\mathrm{E}}\right) \\
H_{\mathrm{O}} \\
H_{\mathrm{E}} \\
\mathrm{PIC}\end{array}$ & $\begin{array}{c}1(1.0000) \\
0.0000 \\
0.0000 \\
0.0000\end{array}$ & $\begin{array}{c}3(2.6486) \\
1.0000 \\
0.6703 \\
0.4488\end{array}$ & $\begin{array}{c}3(2.1978) \\
1.0000 \\
0.5737 \\
0.4415\end{array}$ & $\begin{array}{c}4(1.9413) \\
0.4783 \\
0.4957 \\
0.4581\end{array}$ & $\begin{array}{c}5(2.8556) \\
0.4746 \\
0.6554 \\
0.5887\end{array}$ \\
\hline S336 & $\begin{array}{l}\mathrm{N}\left(A_{\mathrm{E}}\right) \\
H_{\mathrm{O}} \\
H_{\mathrm{E}} \\
\mathrm{PIC}\end{array}$ & $\begin{array}{c}1(1.0000) \\
0.0000 \\
0.0000 \\
0.0000\end{array}$ & $\begin{array}{c}3(2.4615) \\
1.0000 \\
0.6333 \\
0.5587\end{array}$ & $\begin{array}{c}4(2.7692) \\
1.0000 \\
0.6970 \\
0.5683\end{array}$ & $\begin{array}{c}3(2.7128) \\
0.2609 \\
0.6454 \\
0.5587\end{array}$ & $\begin{array}{c}4(3.1947) \\
0.3509 \\
0.6931 \\
0.6390\end{array}$ \\
\hline AIMS007 & $\begin{array}{l}\mathrm{N}\left(A_{\mathrm{E}}\right) \\
H_{\mathrm{O}} \\
H_{\mathrm{E}} \\
\mathrm{PIC}\end{array}$ & $\begin{array}{c}1(1.0000) \\
0.0000 \\
0.0000 \\
0.0000\end{array}$ & $\begin{array}{c}1(1.0000) \\
0.0000 \\
0.0000 \\
0.0000\end{array}$ & $\begin{array}{c}1(1.0000) \\
0.0000 \\
0.0000 \\
0.0000\end{array}$ & $\begin{array}{c}1(1.0000) \\
0.0000 \\
0.0000 \\
0.0000\end{array}$ & $\begin{array}{c}1(1.0000) \\
0.0000 \\
0.0000 \\
0.0000\end{array}$ \\
\hline Mean values & $\begin{array}{l}\mathrm{N}\left(A_{\mathrm{E}}\right) \\
H_{\mathrm{O}} \\
H_{\mathrm{E}} \\
\mathrm{PIC}\end{array}$ & $\begin{array}{c}1.13(1.0669) \\
0.0563 \\
0.0447 \\
0.0360\end{array}$ & $\begin{array}{c}2.63(2.2370) \\
0.8750 \\
0.5975 \\
0.4686\end{array}$ & $\begin{array}{c}3.00(2.3028) \\
0.8438 \\
0.6086 \\
0.4390\end{array}$ & $\begin{array}{c}3.38(2.5000) \\
0.3857 \\
0.5277 \\
0.4696\end{array}$ & $\begin{array}{c}4.38(3.0035) \\
0.4029 \\
0.6069 \\
0.5556\end{array}$ \\
\hline
\end{tabular}

Parameters calculated are: number of alleles $(\mathrm{N})$, effective number of alleles $\left(A_{\mathrm{E}}\right)$, the observed $\left(H_{\mathrm{O}}\right)$ and expected $\left(H_{\mathrm{E}}\right)$ heterozygosities, and the polymorphic information content (PIC). 


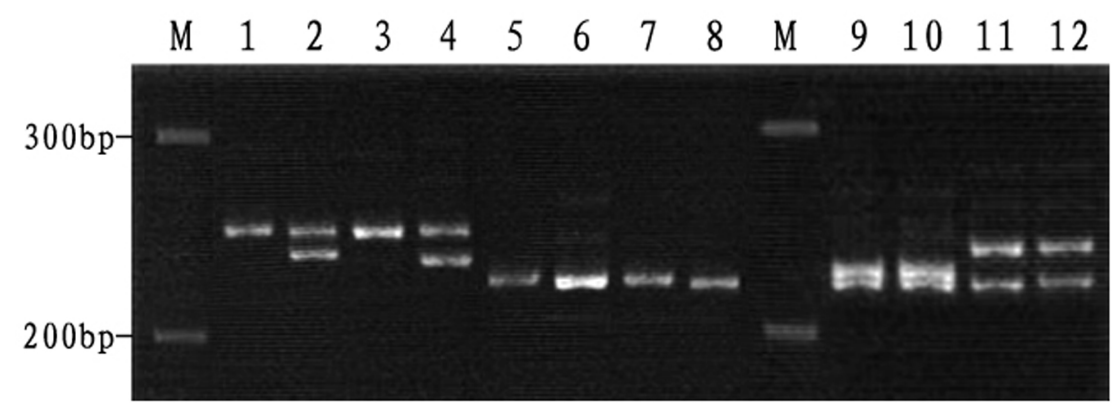

Figure 2. Amplified bands with the SSR primers N391 in parents and their hybrids. Lane $M=100$-bp ladder marker; lanes 1-4 = Argopecten irradians; lanes 5-8=Argopecten purpuratus; lanes 9-12= hybrids.

Table 5. Analysis of molecular variance of the parental groups and the progeny groups using AFLP markers.

\begin{tabular}{lcccc}
\hline & Total variation & Variation between groups and percentage & Variation within groups and percentage & FST \\
\hline PP*BB & 112.145 & $86.183(76.85 \%)$ & $25.962(23.15 \%)$ & 0.769 \\
PB*BP & 40.010 & $10.127(25.31 \%)$ & $29.883(74.69 \%)$ & 0.253 \\
PP*PB & 66.605 & $42.950(64.48 \%)$ & $23.655(35.52 \%)$ & 0.645 \\
PP*BP & 68.606 & $43.447(63.33 \%)$ & $25.159(36.67 \%)$ & 0.633 \\
BB*PB & 73.692 & $44.406(60.26 \%)$ & $29.286(39.74 \%)$ & 0.603 \\
BB*BP & 69.003 & $38.214(55.38 \%)$ & $30.789(44.62 \%)$ & 0.554 \\
\hline
\end{tabular}

Genetic distances and genetic similarity coefficients among groups as estimated by Nei's unbiased measures based on AFLP analysis are presented in Table 6. As expected, the genetic distance between PP and BB (0.5464) was greater than that between the other groups, and was especially greater than the genetic distance between PB and BP (0.0675). The genetic similarity coefficient was consistent with the genetic distances among these groups. The genetic similarity coefficient between $\mathrm{PB}$ and $\mathrm{BP}$ was 0.9347 , which was larger than the genetic similarity within $\mathrm{PB}(0.8727)$ or $\mathrm{BP}(0.8512)$. Thus, the two groups were considered to be one group. Clustering analyses using the UPGMA method also showed that PB and BP were clustered (Figure 3). Moreover, it was found that the genetic distances between hybrids and their maternal parents $(0.2186,0.2320)$ were smaller than those between hybrids and their paternal parents $(0.2701,0.2399)$. Consistently, AMOVA indicated that the genetic variations between hybrids and their maternal parents $(42.950,38.214)$ were somewhat smaller than those between them and their paternal parents $(44.406,43.447)$.

Table 6. Genetic similarity within and between populations and genetic distance between populations by AFLP analysis.

\begin{tabular}{lcccr}
\hline Pop ID & PP & PB & BP & BB \\
\hline PP & 0.8992 & 0.8036 & 0.7867 & 0.5791 \\
PB & 0.2186 & 0.8727 & 0.9347 & 0.7633 \\
BP & 0.2399 & 0.0675 & 0.8512 & 0.7930 \\
BB & 0.5464 & 0.2701 & 0.2320 & 0.8609 \\
\hline
\end{tabular}

On the diagonal is genetic similarity within population; above the diagonal is Nei's genetic identity and below is genetic distance between populations. 

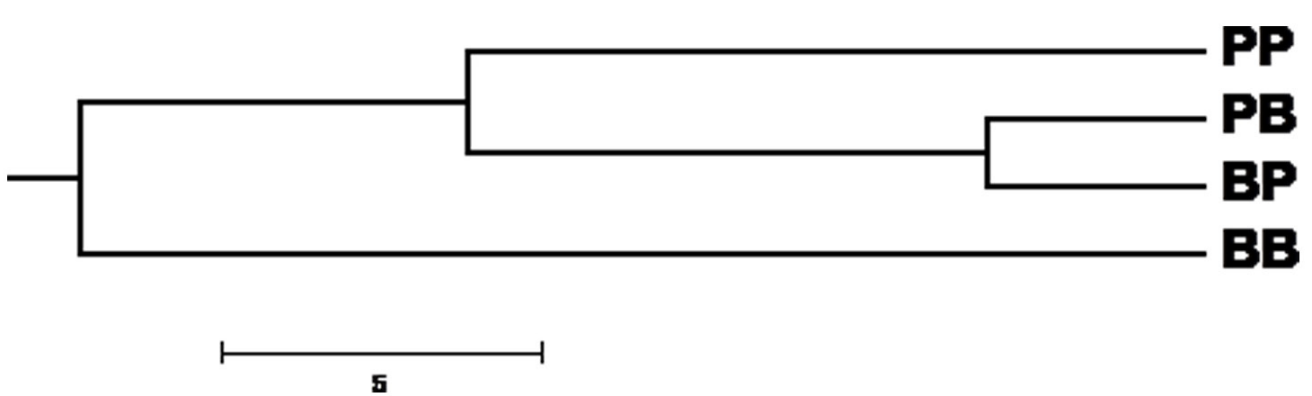

Figure 3. Dendrogram-based Nei's (1978) genetic distance using the UPGMA method by AFLP analysis of Argopecten purpuratus (PP), Argopecten irradians irradians (BB) and their hybrids of A. purpuratus $ᄋ$ x A. $i$. irradians $\hat{\sigma}(\mathrm{PB})$ and $A$. i. irradians + x A. purpuratus $\widehat{\partial}(\mathrm{BP})$.

\section{Genetic diversity of the hybrids and their parents}

The genetic similarity indexes of all four groups are summarized in Table 6. The similarities within groups of $\mathrm{PB}$ and BP were 0.8727 and 0.8512 , respectively, both of which were lower than that within PP (0.8992), and the similarity of BP was also lower than that of BB (0.8609). In contrast, the heterozygosities observed by SSR analysis exhibited a reverse trend. The heterozygosities of hybrids were higher than those of both parents, and those of BP were the highest (Table 4). In addition, the PIC values of the hybrids were estimated at 0.4686 for PB and 0.4390 for BP, respectively, which were both higher than those of PP $(0.0360)$, and lower than those of BB (0.4696) (Table 4). The $h$ indexes and Shannon's information indexes of the four groups based on AFLP analysis were calculated and are shown in Table 7. The $h$ and Shannon's information indexes were both highest in BB and smallest in PP, and were both higher in BP than in PB. Based on these data, the genetic diversities of the hybrids and of BB were found to be at a moderate-to-high level. Furthermore, the genetic diversity and variability of the hybrids was increased compared to the parents, especially to PP.

Table 7. Mean and standard deviation of gene diversity $(h)$ and Shannon's information index within and across four populations studied by AFLP analysis.

\begin{tabular}{lcc}
\hline Populations & $h$ & Shannon's information index \\
\hline PP & $0.0931 \pm 0.1618$ & $0.1429 \pm 0.2374$ \\
PB & $0.1214 \pm 0.1892$ & $0.1798 \pm 0.2716$ \\
BP & $0.1251 \pm 0.1808$ & $0.1891 \pm 0.2640$ \\
BB & $0.1331 \pm 0.1832$ & $0.2020 \pm 0.2663$ \\
Across populations & $0.2886 \pm 0.1797$ & $0.4338 \pm 0.2416$ \\
\hline
\end{tabular}

\section{DISCUSSION}

Hybridization could bring about the recombination of existing genes, which is an important way to increase biodiversity. Hybrid offspring may show good economic traits, or could be unviable, sterile, or have a hybrid disadvantage. Wang et al. (2011) observed that the hybrid offspring of $A$. purpuratus x A. irradians irradians displayed hybrid vigor, which included fast growth. A. purpuratus and A. irradians irradians were introduced from different geographical regions and are distinct in size, life span, and temperature tolerance (Wang et 
al., 2011). AMOVA revealed that the genetic differential index (FST) between these species was 0.769 , and that the genetic variations were significantly different $(\mathrm{P}<0.001)$. This shows that $A$. purpuratus and A. irradians irradians have different genomic constitutions, and could provide a genetic basis for heterosis in their hybrids. The dominance hypothesis and the overdominance hypothesis used to explain heterosis are built on the basis of genetic differences, which guide the heterosis prediction using genetic distance. AFLP and SSR analysis in this study confirmed that the $\mathrm{F} 1$ hybrids of $A$. purpuratus $\mathrm{x}$ A. irradians irradians inherited most genetic material from both parents. The recombination of existing genes was realized in the hybrids. It is possible that the heterosis of the hybrids was attributed to the combination of advantageous genes from both parents and the generation of a dominant or overdominant effect. In addition, the high genetic similarity coefficient (0.9347) and small genetic differentiation $(0.2531)$ between two hybrid groups suggested that the genetic basis of these two hybrid groups was very close. The intuitive performance was their similar phenotypic traits such as shell color patterns and the distribution of their ribs (Wang et al., 2011).

In our study, the results of SSR analysis showed that the hybrids inherited microsatellite markers from two divergent parental genomes and that the heterozygosities were higher in the hybrids than in the parents. The level of heterozygosity might be positively related to economic characteristics. It has been shown that heterozygosities detected using RFLP and other markers are correlated with economic traits in crops (Zhang et al., 1996). In shellfishes, heterozygosities also positively correlate with growth and vitality (Pogson and Zouros, 1994; Hedgecock et al., 1996; Wan et al., 2004). In this study, the hybrids of A. purpuratus x A. irradians irradians, which possess higher heterozygosity compared to their parents, have also been shown to possess strong heterosis in growth traits. Furthermore, in contrast to heterozygosities, the hybrids were less similar than the parents based on AFLP analysis, which also indicated that genetic variation and diversity of hybrids increased. Teng et al. (2005) analyzed the heterosis of scallop populations (C. farreri, P. yessoensis, and their F1 reciprocal hybrids) with the RAPD method, and suggested that the heterosis of the hybrids $C$. farreri ( $(+) \times P$. yessoensis $(\hat{\partial})$ could be attributed to their greater genetic diversity. A reduction in diversity may result in a loss of genetic variation for disease resistance, thus reducing the capability of a population to adapt to new environments (Allendorf and Phelps, 1980). Thus, genetic diversity is important because it may be positively related to genetic variation and enable populations to adapt to environmental change and stress, which is a possible explanation for the heterosis exhibited in the hybrid offspring.

In SSR analysis, all of the alleles in the four populations were observed in parental groups (PP and BB). Conversely, some alleles were not found in F1 hybrids, for example, 15 and 10 alleles that were observed in the BB group were not found in PB and BP groups, respectively. One probable reason was that fewer F1 individuals were used in the experimental analyses, or alternatively, that the parental individuals (PP or $\mathrm{BB}$ ) only partly contributed to the genetic composition of F1 hybrids. Thus, the effective number of parents might have been less than the actual number used in fertilization. AFLP analysis of the hybrid genome also suggested that the inherited AFLP markers in hybrids were not a simple combination of parental specific markers, but that there was some variation. This genetic variation mainly involved the loss of parental AFLP bands and the acquisition of novel bands. In fact, hybridization can bring about genomic changes (Baack and Rieseberg, 2007). Locus variation involving lost bands and the acquisition of novel bands has been reported in some hybrids such as honey bee (Hunt and Page, 1992), hybrids of brown trout x Atlantic salmon (Elo et al., 1997), and the 
hybrids of Haliotis discus hannai x H. discus discus (Wan et al., 2001). Jone et al. (1983) and Yang (1996) suggested that genetic variation mainly occurs in the gene regulatory regions, which have more flexibility to adapt to natural selection than the coding region does. Thus, the genetic variation in hybrids might be due to changes that occur in the gene regulatory region during the combination of parental genetic material. Such variations in the regulatory region would cause allele conversion in the corresponding coding region (Hunt and Page, 1992), and further lead to the transformation of gene expression patterns, which might be one of the possible reasons to explain heterosis.

Considering the genetic composition, it is relatively common that the parental genetic material is asymmetric in hybrids. Dong et al. (1999) analyzed the genetic relationship between Cyprinus carpio singuonensis, $C$. carpio haematopterus, and their hybrids using RAPD, and found that the genetic composition of hybrids was biased towards the male parents. Liu et al. (2006) reported that the genetic relationships between hybrids of $C$. farreri $\mathrm{x}$ Mimachlamys nobilis and their parents were not equal, and that the female parents transferred more genetic loci to their descendants, as observed by ISSR analysis. Genetic analysis based on the RAPD and ISSR markers indicated that the hybrids from C. farreri $\mathrm{x} P$. yessoensis at the early developmental stage inherited parental specific markers, and that the hybrids were genetically closer to their maternal parents (Teng et al., 2005; He et al., 2007). The previous studies showed that the genetic composition of scallop hybrids was almost biased towards the female parents. Wang et al. (2010) demonstrated that maternally biased gene conversion occurred in the hybrids of $C$. farreri + × A. irradians $\hat{O}$, and that this was considered common in Pectinidae. However, in contrast to the speculation of Wang et al. (2010), biased gene conversion was not found in the hybrids of $A$. purpuratus $\mathrm{x}$ A. irradians irradians based on the ITS sequence analysis (Hu et al., 2013). In addition, we have shown through sequence analysis of the 16S rRNA gene that the mitochondrial genome in hybrids of $A$. purpuratus $\mathrm{x}$ A. irradians irradians is maternally inherited (Hu et al., 2013). In the present study, AFLP analysis showed that the hybrids inherited the majority of amplified loci from both parents, and that they were slightly closer to their maternal parents in terms of genetic distance. Taken together, these data suggest that this maternally biased genetic relationship might be caused by involvement of the matrilineal mitochondrion genome. Furthermore, previous genetic analyses performed on scallop hybrids were based on larvae stage materials because viable adult hybrids were not obtained (Teng et al., 2005; Liu et al., 2006; He et al., 2007; Wang et al., 2010). However, hybrid adults from $A$. purpuratus $\mathrm{x} A$. irradians irradians were viable and the hybrid identity was confirmed by AFLP and SSR analyses in this study.

Heterosis is a complex biological phenomenon, which involves interactions between large numbers of related genes owing to the combination of genomes of different origins. Our study reveals the genomic composition and genetic variation of the hybrids of A. purpuratus $\mathrm{x}$ A. irradians irradians, which provides a genetic basis of heterosis in the hybrids. However, we are unable to fully explain heterosis through these data alone, and further study is required to determine the exact genetic mechanism of heterosis, such as the acquisition of more accurate data regarding genomic composition and genetic variation by next generation sequencing technology and through the analysis of hybrid gene networks above the level of gene expression.

\section{Conflicts of interest}

The authors declare no conflict of interest. 


\section{ACKNOWLEDGMENTS}

Research supported by the National Natural Science Foundation of China (\#31270047 and \#31172404), the National Key Technology R\&D Program of China (\#2011BAD13B05 and \#2011BAD45B01), the Earmarked Fund for Modern Agro-industry Technology Research System, Seed Improvement Project of Shandong Province, and the National Infrastructure of Fishery Germplasm Resources.

\section{REFERENCES}

Allendorf FW and Phelps SR (1980). Loss of genetic variation in a hatchery stock of cutthroat trout. Trans. Am. Fish Soc. 109: 537-543.

Baack EJ and Rieseberg LH (2007). A genomic view of introgression and hybrid speciation. Curr. Opin. Genet. Dev. 17: 513-518.

Beck ML and Biggers CJ (1982). Chromosomal investigation of Ctenopharyngodon idella $\mathrm{x}$ Aristichthvs nobilis hybrids. Experientia 38: 319 .

Bower SM, Blackbourn J and Meyer GR (1997). A new and unusual species of Perkinsus pathogenic to cultured Japanese scallops, Patinopecten yessoensis, in British Columbia, Canada. J. Shellfish Res. 16: 333.

Cruz P and Ibarra AM (1997). Larval growth and survival of two catarina scallop (Argopecten circularis, Sowerby, 1835) populations and their reciprocal crosses. J. Exp. Mar. Biol. Ecol. 212: 95-110.

Dall WH (1909). The mollusca and branchiopoda. Report of the dredging operation, 'Albatros' 1891. Bull. Mollusca Comp. Zool. 37: 147-294.

Dong Z, Xia D, Wu T, Yang H, et al. (1999). RAPD analysis of heterosis between xingguo red carp and scattered mirror carp. J. Shang. Fish. Univ. 8: 31-36.

Elo K, Ivanoff S, Vuorinen JA and Piironen J (1997). Inheritance of RAPD markers and detection of interspecific hybridization with brown trout and Atlantic salmon. Aquaculture 152: 55-65.

He B, Yang A, Wang Q, Liu Z, et al. (2007). ISSR analysis of the F1 hybrids of scallop Chlamys farreri $\times$ Patinopecten yessoensis ${ }^{3}$. J. Dalian Fish. Univ. 22: 273-277.

Hedgecock D and Davis JP (2000). Improving pacific oyster brood stock through crossbreeding. J. Shellfish Res. 19: 614-615.

Hedgecock D, McGoldrick DJ, Manahan DT, Vavra J, et al. (1996). Quantitative and molecular genetic analyses of heterosis in bivalve molluscs. J. Exp. Mar. Biol. Ecol. 203: 49-59.

Hu L, Huang X, Mao J, Wang C, et al. (2013). Genomic characterization of interspecific hybrids between the scallops Argopecten purpuratus and A. irradians irradians. Plos One 8: e62432.

Hunt GJ and Page RE (1992). Patterns of inheritance with RAPD molecular markers reveal novel types of polymorphism in the honey bee. Theor. Appl. Genet. 85: 15-20.

Jeffreys AJ, Wilson V and Thein SL (1985). Hypervariable 'minisatellite' regions in human DNA. Nature 314: 67-73.

Jone MA, Strommer JN and Freeling M (1983). Exceptionally high levels of restriction site polymorphism in DNA near the maize ADHI gene. Genetics 105: 733-743.

Liu G, Bao Z, Hu J, Wang S, et al. (2006). ISSR analysis of two species of scallop (Chlamys farreri, C. nobilis) and their intra- and inter-species mating descendants. Period. Ocean Univ. China 36: 71-75.

Nei M (1978). Estimation of average heterozygosity and genetic distance from a small number of individuals. Genetics 89: 583-590.

Pogson GH and Zouros E (1994). Allozyme and RFLP heterozygosities as correlates of growth rate in the scallop Placopecten magellanicus: a test of the associative overdominance hypothesis. Genetics 137: 221-231.

Powell W, Morgante M, Andre C, Hanafey M, et al. (1996). The comparison of RFLP, RAPD, AFLP and SSR (microsatellite) markers for germplasm analysis. Mol. Breed. 2: 225-238.

Roberts S, Romano C and Gerlach G (2005). Characterization of EST derived SSRs from the bay scallop, Argopecten irradians. Mol. Ecol. Notes 5: 567-568.

Sambrook J, Fritsch EF and Maniatis T (1989). Molecular cloning: A laboratory manual. 2nd edn. Cold Springs Harbour Laboratory Press, Cold Springs Harbour, New York.

Schneider S, Roessli D and Excoffier L (2000). Arlequin ver. 2.000: A software for population genetics data analysis. University of Geneva, Geneva.

Song L, Li J, Li H, Cui Z, et al. (2002). The genetic structure and genetic differentiation of the natural population and the 
hatchery stock of Chlamys farreri revealed by RAPD analysis. High Tech. Lett. 12: 83-86.

Teng L, Yang A, Zhao F, Liu Z, et al. (2005). RAPD analysis on the heterosis in reciprocal hybrids of scallop Chlamys farreri $\mathrm{x}$ Patinopecten yessoensis. High Tech. Lett. 6: 97-101.

Vos P, Hogers R, Bleeker M, Reijans M, et al. (1995). AFLP: a new technique for DNA fingerprinting. Nucleic Acids Res. 23: 4407-4414.

Waller TR (1969). The evolution of the Argopecten gibbus stock, with emphasis on the tertiary and quaternary species of eastern North America. J. Paleontol. 43 (Suppl. to No.5, Mem.3): 1-125.

Wan J, Wang X, Pan J, Li B, et al. (2001). RAPD analysis of the genetic change in parent abalone and their hybrids. $J$. Ocean Univ. China 31: 506-512.

Wan J, Bao Z, Zhang Q and Wang X (2004). Comparative studies on the molecular genetic diversities among Haliotis discus hannai, H. discus discus and their hybrids. High Tech. Lett. 10: 93-96.

Wang C, Liu B, Li J, Liu S, et al. (2011). Introduction of the Peruvian scallop and its hybridization with the bay scallop in China. Aquaculture 310: 380-387.

Wang S, Zhang L, Hu J, Bao Z, et al. (2010). Molecular and cellular evidence for biased mitotic gene conversion in hybrid scallop. BMC Evol. Biol. 10: 6.

Wang Y and Guo X (2004). Chromosomal rearrangement in Pectinidae revealed by rRNA loci and implications for bivalve evolution. Biol. Bull. 207: 247-256.

Yan J, Sun H, Fang J, Zhang X, et al. (1999). Study on the technology of crossbreeding abalones Haliotis discus discus and Haliotis discus hannai Ino. Mar. Fish. Res. 20: 35-39.

Yang A, Wang Q, Liu Z and Zhou L (2004). The hybrid between the scallops Chlamys farreri and Patinopecten yessoensis and the inheritance characteristics of its first filial generation. Mar. Fish. Res. 25: 1-5.

Yang J (1996). Mechanism of heterosis: Research of male sterility and heterosis in crop (I). China Agriculture Press, Beijing.

Yeh FC, Yang R and Boyle T (1999). POPGENE (version 1.3.1). Microsoft Window-bases Freeware for Population Genetic Analysis. University of Alberta and the Centre for International Forestry Research, Edmonton.

Zhan A, Bao Z, Wang X and Hu J (2005). Microsatellite markers derived from bay scallop Argopecten irradians expressed sequence tags. Fisheries Sci. 71: 1341-1346.

Zhan A, Hu J, Wang X, Lu W, et al. (2006). A panel of polymorphic EST-derived microsatellite loci for the bay scallop (Argopecten irradians). J. Molluscan Stud. 72: 436-438.

Zhang F, He Y, Liu X, Ma J, et al. (1986). A report on the introduction, seed culture and experimental grow-out of the bay scallop, Argopecten irradians Larmarck. Oceanol. Limnol. Sin. 17: 367-374.

Zhang H, Liu X, Zhang G and Wang C (2007). Growth and survival of reciprocal crosses between two bay scallops, Argopecten irradians concentricus Say and Argopecten irradians irradians Lamarck (1819). Aquaculture 272: S88-S93.

Zhang Q, Zhou Z, Yang G, Xu C, et al. (1996). Molecular marker heterozygosity and hybrid performance in indica and japonica rice. Theor. Appl. Genet. 93: 1218-1224. 